\title{
Presencia de Renilla octodentata Zamponi y Pérez, 1995 (Cnidaria, Pennatulacea, Renillidae) en la Bahía de Valparaíso $\left(33^{\circ} \mathrm{S}-7^{\circ} \mathrm{W}\right)$
}

\author{
Carlos Daniel Pérez \\ Departamento de Ciencias Marinas, Facultad de Ciencias Exactas y Naturales \\ Universidad Nacional de Mar del Plata, Dean Funes 3250, 7600 Mar del Plata, Argentina
}

RESUMEN. Se analiza un ejemplar procedente de las colecciones del Museo Argentino de Ciencias Naturales "Bernardino Rivadavia", perteneciente a la familia Renillidae. Se trata de la especie Renilla octodentata hallada en la Bahía de Valparaíso, Chile, y constituye el primer registro para el Océano Pacífico. El análisis morfométrico comparativo muestra que este ejemplar es aparentemente más grande y pesado que aquéllos del Océano Atlántico.

Palabras claves: nueva cita, Renilla octodentata, Bahía de Valparaíso, Chile.

\section{Presence of Renilla octodentata Zamponi and Pérez, 1995 (Cnidaria, Pennatulacea, Renillidae) in Valparaíso Bay $\left(3^{\circ} \mathrm{S}-7^{\circ} \mathrm{W}\right)$}

\begin{abstract}
One specimen from collections of Argentine Museum of Natural Sciences "Bernardino Rivadavia" was analized, belonging to the Renillidae family. It was Renilla octodentata found in Valparaíso Bay, Chile, and it constitute the first record for Pacific Ocean. Apparently the chilean specimen is bigger and more heavy than the Atlantic's organisms.
\end{abstract}

Key words: new record, Renilla octodentata, Valparaíso Bay, Chile.

Zamponi y Pérez (1995) efectuaron la revisión del género Renilla Lamarck, 1816 para las aguas de la región subantártica del Océano Atlántico Sudoccidental, evidenciando una distribución continua del género Renilla a lo largo de la costa argentina.

De las colecciones del Museo Argentino de Ciencias Naturales "Bernardino Rivadavia" se analizó una muestra con un ejemplar perteneciente a la familia Renillidae Gray, 1860 capturado en aguas chilenas. El análisis pormenorizado de esta muestra indicó la presencia de la especie Renilla octodentata (Fig. 1), cuyos datos de recolección son

\section{Renilla octodentata}

Zamponi y Pérez, 1995

MACN No25436

Fecha de recolección: XII/1941

Dr. Edwyn Reed col., Bahía de Valparaíso, Chile
El hallazgo de esta especie constituye el primer registro para la Provincia peruana (Stuardo, 1964) en aguas del Pacífico Sudoriental, y sustenta el criterio expresado por Broch (1958) sobre la distribución continua de este género a lo largo de las costas americanas. La posibilidad de encontrar Renilla octodentata en aguas del Océano Pacífico Sudoriental indica que esta especie no está restringida al Mar Epicontinental argentino, donde fue hallada por Zamponi y Pérez (1995), sino que presenta una distribución bioceánica (Atlántico-Pacífico).

El hecho de poseer ejemplares de la misma especie procedentes del Océano Atlántico Sudoccidental (Golfo San Jorge, Bahía Camarones y Puerto Deseado), permitió realizar un análisis morfométrico comparativo entre especímenes de ambas zonas (Tabla 1). Si bien el hallazgo de un solo ejemplar no es suficiente para establecer con- 


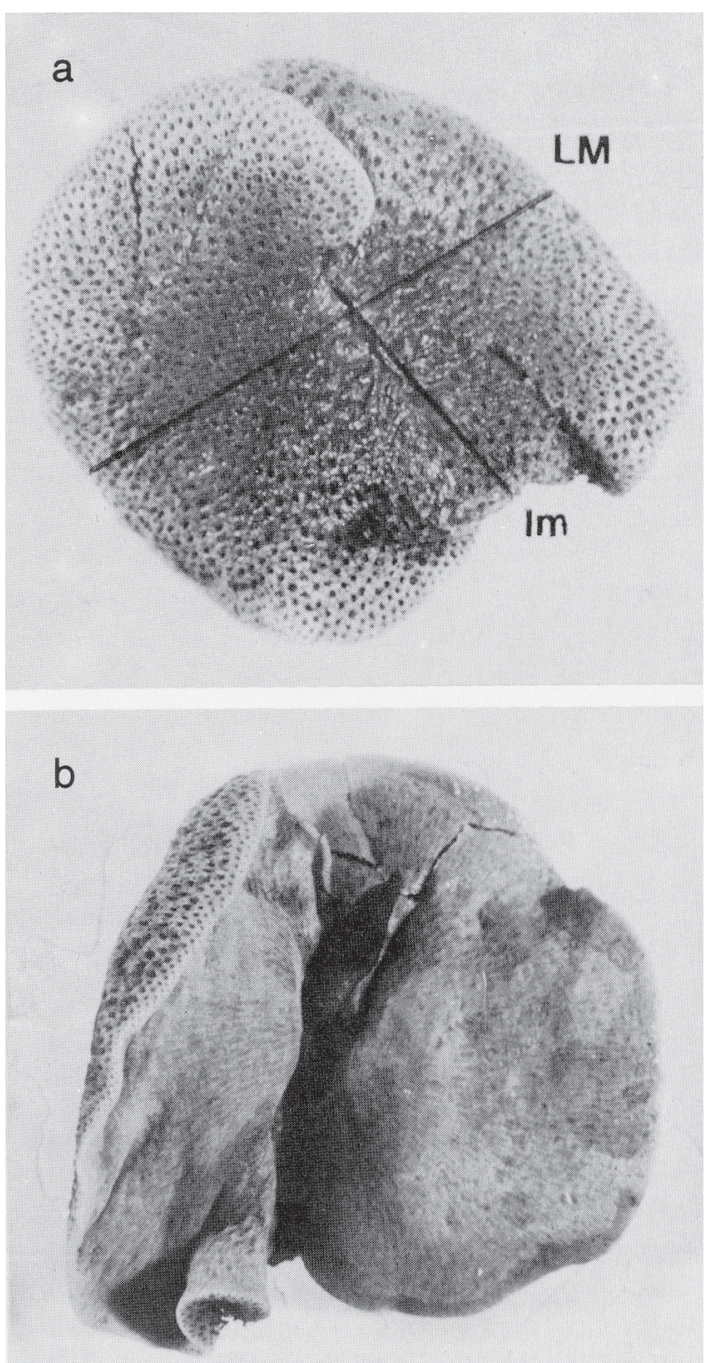

Figura 1. Renilla octodentata: a) vista dorsal, y b) vista ventral (x 1,6). LM: longitud máxima entre los bordes del raquis; lm: longitud del tracto dorsal medio.

Figure 1. Renilla octodentata: a) dorsal view, and b) ventral view (x 1,6). LM: maximum length between the edges of the rachis; Im: maximum length of the medium dorsal tractus.

sideraciones relativas al tamaño de los organismos, se puede destacar que el ejemplar procedente de la Bahía de Valparaíso es aparentemente mayor que el promedio de las tallas de aquellos ejemplares analizados por Zamponi y Pérez (1995), procedentes de aguas del Atlántico Sudoccidental.
Tabla 1. Longitud máxima entre los bordes del raquis (LM) y del tracto dorsal medio (Im) de ejemplares del Atlántico Sur y del de Valparaíso. $\mathrm{S}_{\mathrm{LM}}$ : desviación estándar de $\mathrm{LM} ; \mathrm{S}_{\mathrm{Im}}$ : desviación stándar de $\mathbf{l m}$.

Table 1. Maximum length between the edges of the rachis (LM) and of the medium dorsal tractus (lm) of individuals from the South Atlantic and from Valparaíso. $S_{L M}$ : standard deviation of $\mathrm{LM} ; \mathrm{S}_{\mathrm{Im}}$ : standard deviation of Im.

\begin{tabular}{|l|c|c|c|c|c|}
\hline Renilla octodentata & $\mathrm{n}$ & $\mathrm{LM}(\mathrm{cm})$ & $\mathrm{lm}(\mathrm{cm})$ & $\mathrm{S}_{\mathrm{LM}}$ & $\mathrm{S}_{\mathrm{Im}}$ \\
\hline Atlántico Sur & 8 & 6,8 & 3,09 & 2,34 & 0,44 \\
\hline Bahía de Valparaíso & 1 & 11,0 & 4,80 & - & - \\
\hline
\end{tabular}

Tabla 2. Peso y longitud de un ejemplar del Atlántico Sur y de Valparaíso.

Table 2. Length and weight of an individual from the South Atlantic and one from Valparaiso.

\begin{tabular}{|l|c|c|c|c|}
\hline Renilla octodentata & Peso $(\mathrm{g})$ & $\mathrm{LM}(\mathrm{cm})$ & $\mathrm{Im}(\mathrm{cm})$ & Peso/LM \\
\hline Atlántico Sur & 3,46 & 10,5 & 3,02 & 0,33 \\
\hline Bahía de Valparaíso & 9,52 & 11,0 & 4,80 & 0,86 \\
\hline
\end{tabular}

Asimismo, se observó que el ejemplar procedente de la bahía de Valparaíso es más pesado que los encontrados en el Atlántico Sudoccidental; para tales fines se compararon ejemplares de tallas similares (Tabla 2). Establecida la diferencia de peso entre los ejemplares, se estimó la relación entre el peso y la longitud máxima entre los bordes del raquis (LM), comprobándose también aquí una neta diferencia entre ambos ejemplares (Tabla 2).

Posiblemente si se tuviera la oportunidad de contar con un mayor número de ejemplares, estas observaciones pudiesen ser confirmadas, lo cual será un aporte más al hecho que el aumento de tamaño en relación con el descenso de la temperatura pareciera seguir pautas similares a la regla de Jordan (Margalef, 1977).

\section{AGRADECIMIENTOS}

Se desea expresar el reconocimiento al Dr. Mauricio Zamponi por su colaboración brindada; al Prof. 
Irigoyen, curador del Museo Argentino de Ciencias Naturales "Bernardino Rivadavia", por el préstamo del material estudiado; y al Sr. Abel Berutti, Jefe de Laboratorio de Fotografía de la Universidad Nacional de Mar del Plata, por las fotografías del presente trabajo.

\section{REFERENCIAS BIBLIOGRAFICAS}

Broch, H. 1958. Octocorals. Part I. Pennatularians. Discovery Rep., 29: 245-280.

Recibido el 4 de julio de 1996.

Aceptado el 21 de diciembre de 1996.
Margalef, R. 1977. Ecología. Ediciones Omega, Barcelona, $951 \mathrm{pp}$.

Stuardo, J. 1964. Distribución de los moluscos litorales en Latinoamérica. Bol. Inst. Biol. Mar. de Mar del Plata, 7: 79-91.

Zamponi, M. y C. Pérez. 1995. Revision of the pennatulacean genus Renilla Lamarck, 1916 (Octocorallia, Pennatulacea), with descriptions of two new species from the Subantarctic Region. Miscel-lánia Zoologica, 18: 1-21. 